\title{
Leupaxin Promotes Bladder Cancer Proliferation, Metastasis, and Angiogenesis Through the PI3K/AKT Pathway
}

\author{
Teng Hou ${ }^{a}$ Lijie Zhou ${ }^{a}$ Longwang Wang ${ }^{a, b} \quad$ Gallina Kazobinka \\ Yumao Chen ${ }^{\mathrm{a}}$ Xiaoping Zhang ${ }^{\mathrm{a}}$ Zhaohui Chen $^{\mathrm{a}}$ \\ aDepartment of Urology, Union Hospital, Tongji Medical College, Huazhong University of Science and \\ Technology, Wuhan, bepartment of Urology, The Second Affiliated Hospital of Nanchang University, \\ Nanchang, China
}

\author{
Key Words \\ Lpxn • Bladder cancer • Proliferation • Invasion • Angiogenesis • PI3K/Akt
}

\begin{abstract}
Background/Aims: Leupaxin (LPXN) is a member of the paxillin protein family. Several studies have reported that LPXN regulates cancer development; however, the role of LPXN in bladder cancer remains unknown. Methods: The expression of LPXN in bladder cancer cells and tissues was determined by real-time PCR, western blotting, and immunohistochemistry, respectively. The biological role of LPXN in bladder cancer cell proliferation, invasion, and angiogenesis was explored both in vitro and in vivo. Results: LPXN expression was elevated in bladder cancer tissues and cell lines compared to adjacent non-tumor tissues and normal urothelial cells. High LPXN expression was correlated with large tumor size, advanced tumor stage, and poor survival in bladder cancer patients. Overexpression of LPXN significantly promoted the proliferation, invasion, and angiogenesis of bladder cancer cells, while suppressing LPXN had the opposite effects. The impact on tumor progression was abolished by inhibiting PI3K/ AKT signaling pathway. We further demonstrated that LPXN probably up-regulated S100P via the PI3K/AKT pathway. Conclusions: LPXN may facilitate bladder cancer progression by upregulating the expression of S100P via PI3K/AKT pathway. These results provide a novel insight into the role of LPXN in tumorigenesis and progression of bladder cancer and potential therapeutic target of bladder cancer.

\section{Introduction}

Bladder cancer is one of the most frequently occurring malignant neoplasms in the genitourinary system [1]. Despite some progress in the management of bladder cancer, the prognosis of patients with late-stage diseases remains poor, with about 14 months of median

T. Hou and L. Zhou contributed equally to this work

Dr. Zhaohui Chen

KARGER 
survival time, and about $15 \%$ of long-term disease-free survival [2]. Currently, very little is known about molecular mechanisms triggering the neoplastic transformation of urothelial cells and the progression of bladder cancer. There is urgent need to better understand cancer biology and identify novel molecular pathways involved in disease progression for bladder cancer therapy.

Leupaxin (LPXN), a member of the paxillin protein family, is characterized by the presence of protein-protein interaction domain structure composed of LIM domains and LD motifs [3]. The LPXN gene is located in chromosome 11q12.1 and encodes 386 amino acids [4]. It was originally known as a multifunctional adaptor protein preferentially expressed in hematopoietic cells [3]. Later, LPXN was identified as a specific regulator in osteoclasts during the formation of adhesion zones. Disruption of LPXN's association with the podosomal signaling complex results in the inhibition of osteoclast migration and osteoclastic resorption [5]. In addition, LPXN is enriched in vascular smooth muscle and functions as a serum response factor to induce smooth muscle marker gene expression [6]. A growing body of literature suggests that LXPN may be a critical regulator in the development of several human malignancies. For example, LXPN was expressed in prostate cancer and contributed to regulating cancer cell adhesion and invasion [7]. Additionally, LPXN reportedly increased motility and invasiveness of prostate cancer cells through mediation of p120catenin [8]. Moreover, upregulated LPXN expression was detected in mammary carcinoma, and LPXN could induce transcriptional activity of estrogen receptor $\alpha$, which may promote the progression and invasion of breast cancer cells [9]. In acute myeloid leukemia, LPXN interacts with its fusion partner ETV6 to enhance cell proliferative response and migration by enhancing the response to G-CSF and CXCL12 [10]. These findings indicate that LPXN may function as an oncogene in the progression of human cancers. However, the role of LPXN and its underlying molecular mechanism in the bladder cancer progression are largely unknown.

In this study, we examined the expression of LPXN in bladder cancer cell lines and human bladder cancer tissue. The expression of LPXN and its correlation with tumor stage, tumor size and clinical prognosis was also investigated. Moreover, we attempted to identify the molecular mechanism via which LPXN promoted bladder cancer proliferation, metastasis, and angiogenesis. Our findings suggest that LPXN may be a promising molecular target for the therapy of bladder cancer.

\section{Materials and Methods}

\section{Cell culture}

Bladder cancer cell lines BIU-87, T24, J82, UM-UC-3, EJ, TCCSUP, RT4, and human uroepithelial cell SVHUC-1 were purchased from the Cell Bank of the Chinese Academy of Sciences (Shanghai, China). Primary normal bladder urothelial cells (NBUCs) cultures and SV-HUC-1 were established as described previously [11]. The cell lines BIU-87, T24, J82, UM-UC-3, EJ, and TCCSUP were routinely maintained in RPMI 1640 medium (Gibco, USA), while the RT4 cell line was cultured in McCoy's 5a medium supplemented with $10 \%$ fetal bovine serum (FBS) (Gibco, USA). SV-HUC-1 cells was grown in F12K medium supplemented with $10 \%$ FBS.

\section{Clinical samples}

A total of 196 paraffin-embedded specimens of bladder cancer tissues were obtained from The Second Affiliated Hospital of Nanchang University and used for immunohistochemistry (IHC). The clinical stage of patients was determined according to the American Joint Committee on Cancer (AJCC)'s classification system on TNM staging. Sample collections were performed after receiving approval from the institutional ethics review committee of The Second Affiliated Hospital of Nanchang University. In addition, 60 pairs of snap-frozen bladder cancer and normal urothelium samples were obtained and frozen immediately in liquid nitrogen after surgical removal and stored at $-80^{\circ} \mathrm{C}$ until use. 


\section{EdU Labeling}

Cells were plated on coverslips, and incubated for $3 \mathrm{~h}$ with 5-ethynyl-2'-deoxyuridine (EdU, RiboBio; R11053), and treated with ApolloR reaction cocktail according to the manufacturer's instructions. Images were acquired under a fluorescent microscopy (Olympus, Japan).

\section{Flow cytometry analysis}

Cells were harvested by trypsinization, washed in ice-cold PBS, and fixed with $75 \%$ ethanol, followed by incubation with Bovine pancreatic RNAase $\left(2 \mu \mathrm{g} / \mathrm{ml}\right.$, Sigma-Aldrich; USA) at $37^{\circ} \mathrm{C}$ for $30 \mathrm{~min}$. Then, the cells were incubated in propidium iodide $(20 \mathrm{mg} / \mathrm{ml}$, Sigma-Aldrich; USA) for $20 \mathrm{~min}$ at room temperature. Cell cycle was analyzed by using a BD LSRFortessa X-20 instrument (BD Biosciences, CA, USA).

\section{Transwell invasion assay}

Cell invasion was examined using the transwell matrigel invasion assay. After pretreated, cells were suspended in serum-free DMEM medium and then seeded in the cell culture insert chambers precoated with matrigel (BD Biosciences, San Jose, CA, USA). Culture medium supplemented with 10\% FBS was added to the well, and the cells were then incubated for $24 \mathrm{~h}$. Cells remaining on the top surface were removed and cells migrated to the lower surface of the membrane were fixed with $4 \%$ paraformaldehyde and stained with $0.1 \%$ crystal violet (Sigma, St. Louis, MO, USA). A light microscope was then used to count the number of cells in five randomly selected areas.

\section{Plasmids, lentiviral infection, and transfection}

Human LPXN cDNA was amplified by PCR and cloned into GV358 lentiviral vector (GeneChem, Shanghai, China). Oligo of LPXN shRNAs was synthesized and inserted in GV248 vector Genechem Co.,Ltd (GeneChem, Shanghai, China). Stable cell lines that expressed LPXN or LPXN-shRNAs were selected for 10 days with $0.5 \mathrm{mg} / \mathrm{ml}$ puromycin.

\section{Quantitative real-time PCR ( $q R T-P C R)$}

Total RNA of cell lysates and tissues was extracted in accordance with TRIzol solution (Invitrogen, California, USA). cDNA was generated from 1 ug of each RNA sample and reverse transcribed using a RevertAid First Strand cDNA Synthesis kit (Thermo, Massachusetts, USA). All of the quantitative real-time PCR experiments were performed using an StepOne Plus real-time PCR system (Life Technologies, Carlsbad, CA, USA). The sequences of primers of LPXN are as follows: forward, 5'-CTGGAATGGGAGACCTGTTG-3', reverse, 5'-CCCTGGATTGTGTGGGTATG-3'. GAPDH was used as an internal control.

\section{Western blotting}

Cell lysates and tissues were harvested using RIPA lysis buffer, and cleared by centrifugation at $4^{\circ} \mathrm{C}$. A Bradford assay (Thermo Scientific, Massachusetts, USA) was used to determine cell protein concentrations. The proteins were then transferred onto polyvinylidene difluoride membranes and immunoblotted with primary antibodies: LPXN, S100P, E-cadherin, N-cadherin, Vimentin, Fibronectin (1:1000; Abcam, USA), p-PI3K(Tyr458/Tyr199), PI3K, p-AKT(Thr 308), p-AKT(Ser 473), AKT, p-GSK-3ß(Ser9), p-ERK1/2(Thr202/ Tyr204), and ERK1/2 (1:1000; Cell signaling, Boston, MA, USA). After incubation with a secondary antibody, the blots were visualized by enhanced chemiluminescence (Amersham). $\alpha$-tubulin was used as a loading control.

\section{Immunohistochemistry}

The paraffin-embedded specimens were cut into 4- $\mu \mathrm{m}$ thick section. Sections were deparaffinized with xylenes and rehydrated, and were then treated with $3 \%$ hydrogen peroxide in methanol to quench the endogenous peroxidase activity, followed by incubation with $1 \%$ fish skin gelatin to block the nonspecific binding. Tissue sections were incubated with anti-LPXN, anti-S100P, anti-CD31, and anti-Ki67 antibodies (Abcam, Cambridge, USA; $1: 150$ ) at $4^{\circ} \mathrm{C}$ overnight, and incubated with a biotinylated secondary antibody (Abcam, Cambridge, USA), followed by further incubation with 3, 3-diaminobenzidine tetrahydrochloride (DAB) and counterstain with haematoxylin. Evaluation of immunohistochemistry (IHC) was performed by two independent observers who were blind to the clinical data of the patients. The degree of immunostaining was reviewed and scored separately by two independent pathologists, based on both the proportion of 
Hou et al.: LPXN Regulates BC Progression via PI3K/AKT

positively stained tumour cells and the intensity of staining. The intensity of staining were graded as 0 (no staining), 1 (weak, light yellow), 2 (moderate, yellowish brown) and 3 (strong, brown). The scores of the extent of immunoreactivity ranged from 0 to 4 , according to the percentage of cells that exhibited positive staining in each microscopic field of view (0, negative; $1,<10 \% ; 2,10 \%-50 \% ; 3,50 \%-80 \% ; 4,80 \%-100 \%)$. The final score was obtained by multiplying the proportion of positive cells and the staining intensity score. A final score of 0-3 was considered as negative, and a final score of 4-12 was considered as positive.

\section{HUVEC tube formation assay}

Briefly, $200 \mu \mathrm{l}$ of precooled Matrigel (Collaborative Biomedical Products) was pipetted into each well of a 24-well plate and polymerized for 30 min at $37^{\circ} \mathrm{C}$. HUVECs $\left(5 \times 10^{4}\right)$ in $200 \mu \mathrm{l}$ conditioned medium were added to each well and incubated at $37^{\circ} \mathrm{C}$ in $5 \% \mathrm{CO}_{2}$ for $20 \mathrm{~h}$. The capillary tube structure was photographed under a bright-field microscope, and quantified by measuring the total length of the completed tubes. Each condition was assessed at least in triplicate.

\section{Anchorage-independent growth ability assay}

The cells were trypsinized and counted. $5 \times 10^{3}$ cells were mixed with complete medium containing $0.3 \%$ agar (Sigma-Aldrich; USA) on 6 -well plate, followed by plating on top of a bottom layer with $1 \%$ agar completed medium mixture. After 10 days incubation, viable colonies larger than $0.1 \mathrm{~mm}$ in diameter were scored.

\section{Tumour xenografts}

All experimental procedures were approved by the Institutional Animal Care and Use Committee of Tongji Medical College of Huazhong University of Science and Technology. BALB/c nude mice (5-6 weeks old) were purchased from the Center of Experimental Animal of Tongji Medical College of Huazhong University of Science and Technology and randomized into groups. For tumor formation assay, $5 \times 10^{6}$ cells was injected subcutaneously into the flanks of the mice. Tumor volume $\left(\mathrm{mm}^{3}\right)=$ length $\times$ width $^{2} \times 0.5$. Mice were sacrificed after 30 days, and the tumors were examined using an IVIS imagining system (Caliper).

\section{Statistical analysis}

All statistical analyses were carried out using SPSS 16.0 software (SPSS, Inc., Chicago, IL, USA). Unpaired Student's t tests were used for normally distributed data and nonparametric Mann-Whitney U-tests were used for non-normally distributed data to compare central tendencies. The correlation between LPXN expression and clinicopathologic features was analyzed using chi-square test or Fisher's exact test. Correlation coefficients were calculated by the Spearman rank correlation test. Survival curves were plotted by the Kaplan-Meier method and the log-rank test was utilized to compare the survival rates between groups with varying LPXN expression levels. P $<0.05$ was considered statistically significant. Data are expressed as mean \pm SD from at least 3 independent experiments.

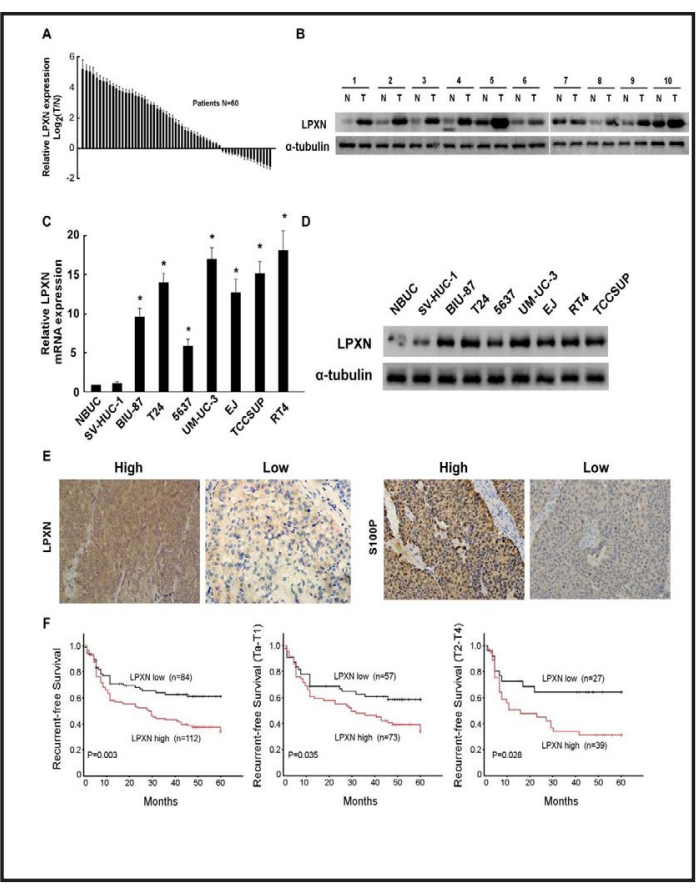

Fig. 1. LPXN expression is upregulated in bladder cancer. (A) LPXN mRNA expression in 60 pairs of bladder cancer and adjacent non-tumor tissues. (B) The protein expression of LPXN in 10 pairs of randomly selected tumor (T) and adjacent nontumor tissues (N). (C-D) LPXN mRNA and protein in normal bladder urothelial cells (NBUCs), human uroepithelial cells (SV-HUC-1), and bladder cancer cell lines (BIU-87, T24, 5637, UM-UC-3, EJ, TCCSUP, and RT4). (E) Representative images of IHC for LPXN and S100P in tissues from bladder cancer patients. 400X. (F) Recurrent-free survival curves in relation to the LPXN status in 196 bladder cancer patients, in 130 patients with Ta-T1 tumor, and in 66 patients with T2-T4 tumor. 


\section{Results}

\section{Expression of LPXN in bladder cancer tissues and cell lines}

In bladder cancer tissue collected from patients, the increased LPXN mRNA expression was found in $73.3 \%$ (44/60) of the cases, compared to that in adjacent non-tumor tissue (Fig. 1A). Consistently, western blot analysis showed that LPXN expression was upregulated in bladder cancer tissue (Fig. 1B). Additionally, we analyzed LPXN mRNA expression in 7 bladder cancer cell lines and normal urothelial cell lines, respectively. LPXN expression was significantly increased in bladder cancer cells in comparison to NBUCs and normal urothelial cells (Fig. 1C and 1D).

\section{LPXN expression was associated with advanced clinical features in bladder cancer}

We investigated whether the levels of LPXN expression is associated with any clinical features of bladder cancer patients. The expression of LXPN was associated with advanced tumor stage ( $\mathrm{T}$ classification) $(\mathrm{P}=0.026)$ as well as the increased tumor size $(\mathrm{P}=0.029)$ (Table 1). There was no significant association with gender, age and histologic tumor grade. Kaplan-Meier analysis showed that the recurrence-free survival time in patients with higher LPXN expression was reversely correlated with LPXN expression (Fig. 1F). Univariate and Multivariate analysis using the Cox proportional hazards model also revealed that LPXN expression was an independent predictor of recurrence-free survival in bladder cancer patients $(\mathrm{P}=0.004$ and $\mathrm{P}=0.002$, respectively) (Table 2$)$.

\section{LPXN expression was closely correlated with S100P expression}

Previous research has established the role of S100P as a urothelial cell marker, both in normal urothelial cells and urothelial cell carcinoma. S100P can also modulate the cytoskeleton and focal adhesion in cancer cells,increase cell proliferation, invasion and migration [12-14]. An immunohistochemistry study was performed to assess whether S100P level was correlated with LXPN expression in bladder cancer. The representative immunostaining of LPXN and S100P in bladder cancer tissues is shown in Fig. 1E. Statistical analysis revealed that LPXN and S100P expression levels were significantly correlated (Kappa $=0.213, \mathrm{P}=0.003$, Table 3).

Table 1. Correlation between LPXN expression and clinicopathological characteristics. $\mathrm{T}$ classification: the anatomic extent of cancer for the tumor (T) of the AJCC TNM staging system.

\begin{tabular}{llllc}
\hline Characteristic & No. & \multicolumn{2}{c}{ LPXN expression } & $P$ \\
& & Low & High & \\
\hline Sex & 121 & 51 & 70 & 0.799 \\
$\quad$ Male & 75 & 33 & 42 & \\
$\quad \begin{array}{l}\text { Female } \\
\text { Age }(y)\end{array}$ & & & & 0.719 \\
$\quad \leq 60$ & 92 & 37 & 55 & \\
$\quad>60$ & 104 & 47 & 63 & \\
$\quad$ Tumor grade & & & & 0.355 \\
$\quad$ Low & 117 & 47 & 70 & \\
$\quad$ High & 79 & 37 & 42 & \\
Tumor size & & & & 0.029 \\
$\quad<3 \mathrm{~cm}$ & 143 & 68 & 75 & \\
$\quad 23 \mathrm{~cm}$ & 53 & 16 & 37 & \\
$\quad$ T classification & & & & 0.026 \\
$\quad$ Ta,T1 & 130 & 63 & 67 & \\
$\quad$ T2-T4 & 66 & 21 & 45 & \\
Total NO. of patients & 196 & 84 & 112 & \\
\hline
\end{tabular}

Table 2. Univariate and multivariate analysis of recurrence-free survival in patients with bladder cancer. $\mathrm{T}$ classification: the anatomic extent of cancer for the tumor (T) of the AJCC TNM staging system.

\begin{tabular}{lllll}
\hline Prognostic variables & Univariate analysis & & Multivariate analysis & \\
& Hazard ratio $(95 \% \mathrm{CI})$ & $P$ & Hazard ratio $(95 \% \mathrm{CI})$ & $P$ \\
\hline Sex (M vs F) & $0.701(0.452-1.089)$ & 0.114 & & \\
Age (>60 vs $\leq 60)$ & $1.030(0.678-1.565)$ & 0.889 & & \\
Tumor grade (High vs Low) & $1.151(0.757-1.751)$ & 0.510 & & \\
Tumor size ( $\geq 3 \mathrm{~cm}$ vs $<3 \mathrm{~cm})$ & $1.806(0.964-3.383)$ & 0.018 & & \\
T classification (T2-4 vs T1,Ta) & $2.028(0.679-3.836)$ & 0.016 & & \\
LPXN (high vs low) & $1.868(1.223-2.851)$ & 0.004 & $2.016(1.304-3.117)$ & 0.002 \\
\hline
\end{tabular}


LPXN promoted cell proliferation, migration, invasion, and angiogenesis in bladder cancer cell lines

To determine the role of LPXN in bladder cancer development, we employed a lentivirus-based expression system to overexpress or suppress LPXN in bladder cancer cells. Western blotting results revealed that LPXN expression level was significantly increased in cells with LPXN-lentivirus infection (Fig. 2A). We observed that overexpressing LPXN markedly increased the proliferation rate in both EJ and T24 cells (Fig. 2B). Coincidently, flow cytometry assay revealed that LPXN overexpression significantly elevated the percentage of S phase (Fig. 2C). The results of transwell assay showed that LPXNtransduced cells exhibited a significantly increased invasive ability (Fig. 2D). Moreover, the HUVEC tube formation assay showed the abilities of bladder cancer cells to induce HUVEC tube formation was markedly increased in LPXN-overexpressing cells (Fig. 2E). In agreement with these results, we observed that silencing of LPXN expression inhibited cell proliferation, invasion and angiogenesis (Fig.s 3A-3E). These in vitro results further supported the role of LXPN in promoting the aggressiveness of bladder cancer cells.

\section{LPXN promoted tumorigenicity of bladder cancer}

We further evaluated the effect of LPXN on the tumorigenic activity of bladder cancer cells. The overexpression of LPXN significantly increased the anchorage-independent growth ability of EJ and T24 cells in soft agar, while silencing of LPXN decreased this ability (Fig. 4A). In vivo study showed that the tumours arising from LPXN-overexpressing cells were larger and heavier than the vector-control tumors (Fig. 4B-4D). Immunohistochemistry staining showed that LPXN-overexpressing tumors exhibited higher Ki67 proliferation index and increased microvascular density (MVD), whereas LPXN suppression had the opposite effect (Fig. 4E). Collectively, these results indicated that LPXN contributed to the tumorigenicity of bladder cancer cells in vivo.

Table 3. Correlation between LPXN and S100P expression in bladder cancer patients.

\begin{tabular}{|c|c|c|c|c|}
\hline \multirow{2}{*}{\multicolumn{2}{|c|}{$\begin{array}{l}\text { LPXN } \\
\text { expression }\end{array}$}} & \multicolumn{2}{|c|}{ S100P expression } & \multirow{2}{*}{$p$ Value } \\
\hline & & Low & High & \\
\hline Low & 84 & 52 & 32 & \multirow[t]{2}{*}{0.003} \\
\hline High & 112 & 45 & 67 & \\
\hline
\end{tabular}

Fig. 2. Up-regulation of LPXN promotes bladder cancer cell aggressiveness in vitro. (A) Western blotting analysis of LPXN expression in EJ and T24 cells stably expressing LPXN; $\alpha$-tubulin was used as a loading control. (B) Representative micrographs (left panel) and quantification (right panel) of EdU incorporation in the indicated bladder cancer cells. DAPI was used as a DNA/ nuclear stain. (C) Flow cytometric analysis showing the percentages of LPXN-overexpressing cells at different phases of the cell cycle. (D) Representative pictures (left panel) and quantification (right

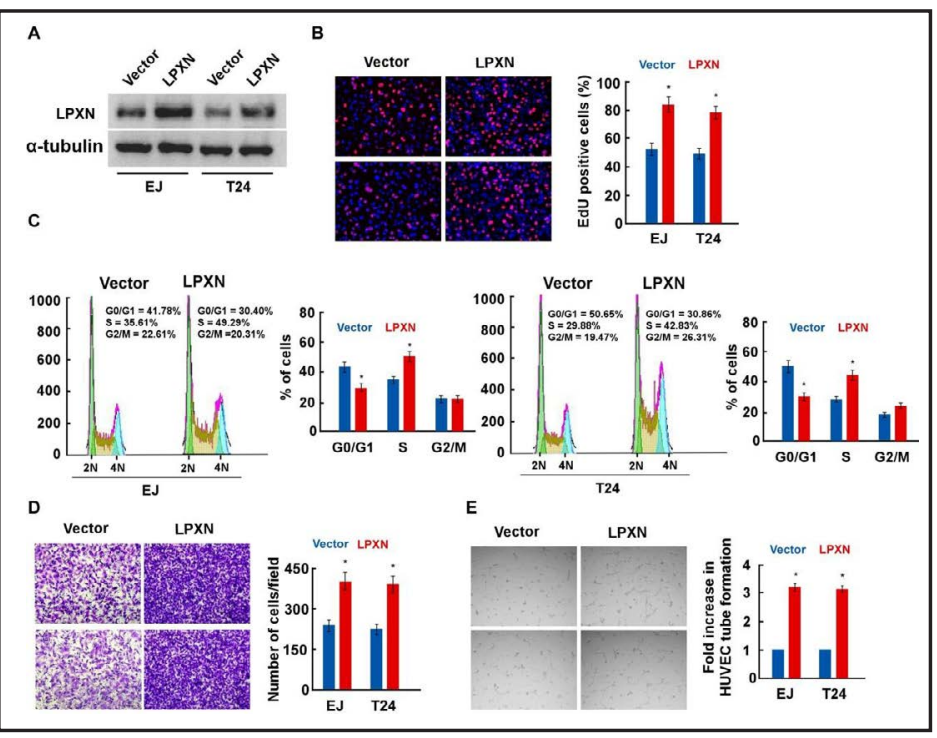
panel) of invaded cells were analyzed using a transwell Matrigel assay. (E) Representative images (left panel) and quantification (right panel) of HUVECs cultured on matrigel-coated plates with conditioned medium from LPXN-overexpressing cells. Bar graphs show the statistical analysis of three independent experiments $\left({ }^{*} \mathrm{P}<0.05\right)$. 
Fig. 3. Downregulation of LPXN inhibits the aggressiveness of bladder cancer cells. (A) Western blotting analysis of LPXN expression in EJ and T24 cells stably silencing LPXN; $\alpha$-tubulin was used as a loading control. (B) Representative micrographs (left panel) and quantification (right panel) of EdU incorporation in the indicated bladder cancer cells. DAPI was used as a DNA/ nuclear stain. (C) Flow cytometric analysis showing the percentages of LPXN-knockdown cells at different phases of the cell cycle. (D) Representative pictures (left panel) and quantification (right panel) of invaded cells were analyzed using a transwell Matrigel assay. (E) Representative images (left panel) and quantification (right panel) of HUVECs cultured on matrigel-coated plates with conditioned medium from LPXNknockdown cells. Bar graphs show the statistical analysis of three independent experiments $(* \mathrm{P}<0.05)$.

Fig. 4. LPXN overexpression suppresses the tumorigenicity of bladder cancer in vitro. (A) Representative micrographs (left) and quantification (right) of colonies formed by the indicated cells, determined by anchorageindependent growth ability assays. (B) Representative images of the tumors from Xenograft model in nude mice. (C) Tumor volumes were measured on the indicated days. (D) Tumor weights of all mice in each group. (E) Immunohistochemical staining demonstrated the expression of Ki67, and CD31 in the indicated tissues. Data are mean \pm SD of three independent experiments. $* \mathrm{P}<0.05$.
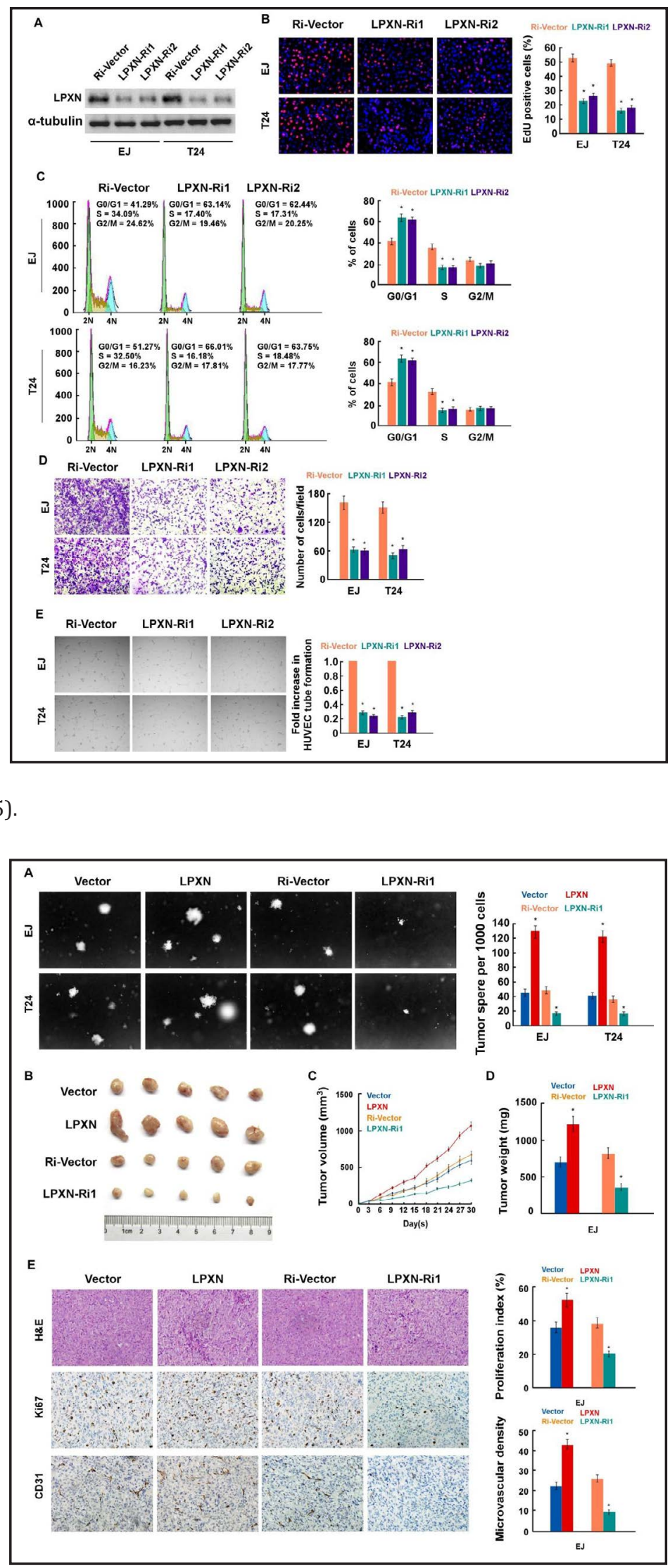
Fig. 5. LPXN regulates the PI3K/ AKT signaling pathway in bladder cancer. (A) GSEA plot showing that LPXN expression negatively correlated with PI3K/AKT, and focal adhesion related gene signatures. (B) Protein levels of PI3K, p-PI3K, AKT, p-AKT, ERK1/2, p-ERK1/2, epithelial and mesenchymal makers, and S100P were shown in indicated cells. (C) LPXN-overexpression cells and vector were treated with PI3K inhibitor LY294002, and protein levels of PI3K, p-PI3K, AKT, p-AKT, ERK1/2, p-ERK1/2, epithelial and mesenchymal makers, and S100P were compared in indicated cells. (D) Western blotting analysis of the expression levels of the indicated proteins in the indicated cells. $\alpha$-tubulin was used as a loading control. (E) Quantification of EdU positive cells in the indicated bladder cancer cells. (F) Quantification of invaded cells were analyzed using a transwell

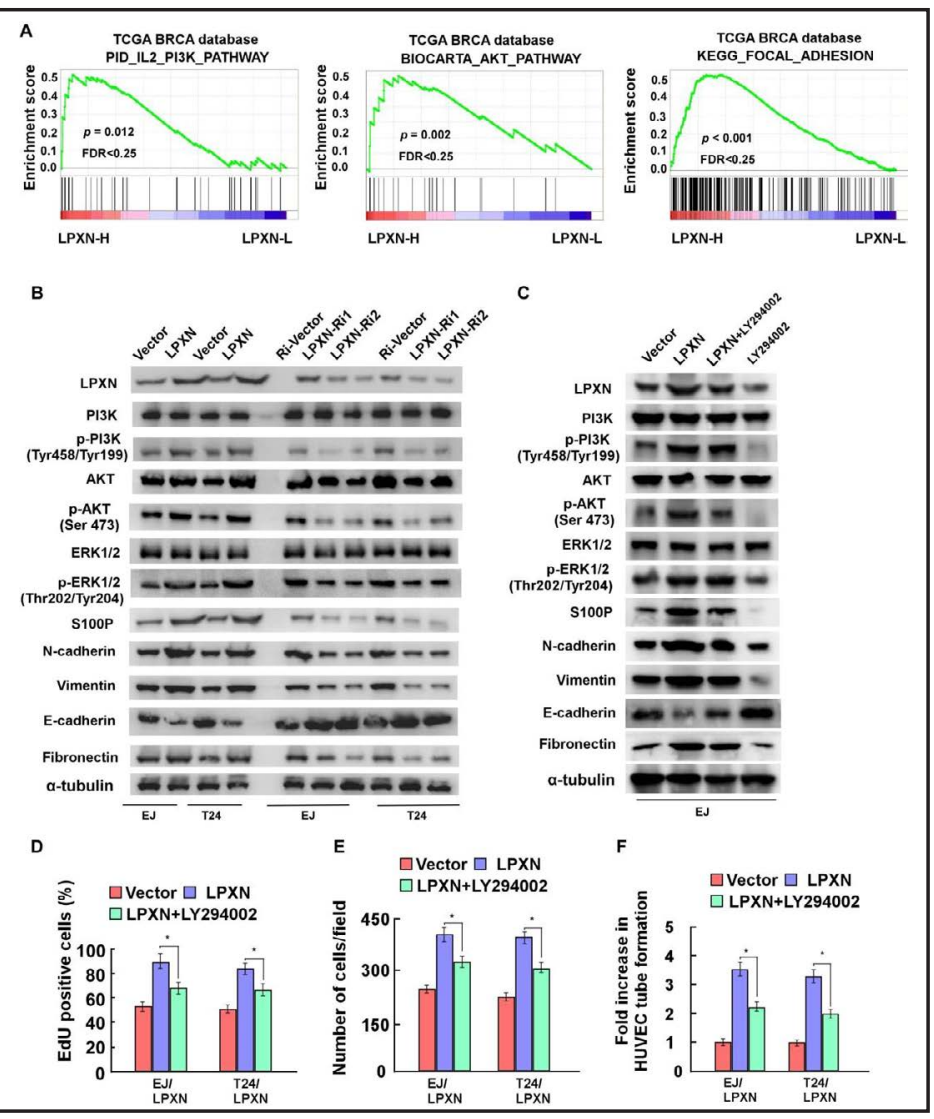
Matrigel assay. (G) Quantification of tubule formation by HUVECs cultured in Matrigel-coated plates with conditioned media from the indicated bladder cancer cells. Each bar represents the mean $\pm \mathrm{SD}$ of three independent experiments. ${ }^{*} \mathrm{P}<0.05$.

\section{LPXN increased S100P expression and induced activation of PI3K/AKT pathway in bladder} cancer

To explore the molecular mechanisms by which LPXN faciliated proliferation, migration, invasion, and angiogenesis in bladder cancer cells, we examined the expression of relevant proteins in the PI3K/AKT pathway. The Gene Set Enrichment Analysis (GSEA) data showed that LPXN upregulation was associated with the activation of the PI3K/AKT signaling pathway and focal adhesion genes (Fig. 5A). Overexpression of LPXN caused a significant increase in PI3K (Tyr458, Tyr199), AKT (Ser473), and ERK1/2(Thr202/Tyr204) phosphorylation. In contrast, phosphorylation of PI3K, AKT, and ERK1/2 was not observed in LPXN-knockdown cells. Additionally, S100P expression was obviously correlated with LPXN level (upregulated in LPXN overexpressing cells and down-regulated inLPXN knockdown cells; Fig. 5B). Moreover, upregulation of N-cadherin, vimentin and fibronectin, and down-regulation of E-cadherin were observed in LPXN overexpressed bladder cancer cells. Conversely, decreased $\mathrm{N}$-cadherin, vimentin, and fibronectin expression and increased of E-cadherin were detected in LPXN silenced cancer cells (Fig.5B).

Consistent with these results, we found that PI3K inhibitor (LY294002) blocked the effect of LPXN-overexpression on protein expression and bladder cancer cell proliferation, migration and angiogenesis (Fig. 5C-5F). Interestingly, the expression of S100P was also decreased following PI3K-specific inhibitor (Fig. 5C), which suggested that LPXN regulated S100P via the PI3K/AKT pathway. Together, these results implicated the regulatory role of LPXN in PI3K/AKT activation and the aggressiveness of bladder cancer cells. 


\section{Discussion}

In the present study, we found that the focal adhesion protein LPXN was an oncoprotein in bladder cancer progression. LPXN expression was associated with advanced tumor stage, lymph node metastasis, and unfavorable clinical prognosis in bladder cancer patients. Moreover, we identified that LPXN facilitated the proliferation, migration, and invasion of bladder cancer cells both in vivo and in vitro, while shRNA-mediated LPXN knockdown decreased the bladder cancer cell proliferation, migration and invasion abilities. In addition, our data demonstrated that these effects of LPXN overexpression were mediated via PI3K/ AKT signaling pathway. It can thus be suggested that LXPN is a crucial regulator in bladder cancer development. Our findings may provide potential therapeutic targets for bladder cancer.

Focal adhesion kinase (FAK) is a non-receptor tyrosine kinase that plays crucial roles in integrin-mediated signalings and cell surface receptor-mediated signal transduction [15]. Previous studies have demonstrated that FAK frequently participates in wide range of essential processes for cancer pathogenesis, including cell adhesion, apoptosis, proliferation, metastasis, and angiogenesis [16]. As a FAK family member, LPXN has been reported to play an essential role in human cancer development $[8,9]$. Early study showed that LPXN could form a signaling complex with protein tyrosine kinases Pyk2, c-Src, and the cytosolic protein tyrosine phosphatase-proline-, glutamate-, serine-, and threonine-rich sequence to modulate prostate cancer cell migration [17]. In another study, Dierks et al. demonstrated that LPXN stimulated prostate cancer cell adhesion and migration through regulation of the phosphorylation of the actin-binding protein caldesmon [18]. In hepatocellular carcinoma (HCC), LPXN contributes to HCC cell proliferation and cell-cycle progression via interacting with $\beta$-catenin and enhance its transcriptional activity [19]. The current study found that LPXN enhanced malignant phenotype including proliferation, migration, and invasion of bladder cancer cells. of the above findings provide compelling evidence to support the LXPN role in human malignancies.

Of note, the prognostic role of FAK has been well studied in cancers. For example, upregulated FAK expression was correlated with poor prognosis and tumor dissemination in hypopharyngeal cancer patients [20]. Similarly, high FAK expression in triple-negative breast cancer was associated with lymphovascular invasion and shorter overall and progression free survival [21]. However, the prognostic role of LPXN in bladder cancer patients has never been investigated. In the present study, we found that LPXN expression was correlated with poor prognosis in bladder cancer. In addition, there was a close correlationship between LPXN expression and tumor stage and size. To our knowledge, the present study is the first reportto offersome important insights into of the predictive value of LXPN as a tumor marker for the outcome of bladder cancer patients.

PI3K/AKT signaling plays a pivotal role in cancer development and progress. The activation of PI3K/AKT signaling facilitates bladder carcinogenesis and cancer progression [22]. However, its relationship with other molecular alterations observed in bladder cancer has not been fully understood. We demonstrated that LPXN promotes the activation of PI3K/AKT signaling, tumorigenicity and progression in bladder cancer. Additionally, our data suggest that the expression of S100Pis positively correlated with LPXN expression in bladder cancer tissues and cells viaPI3K/AKT pathway. Our results are further supported by Li and colleagues [23], who observed that the expression of LASP-1, a focal adhesion adaptor protein, was closely related with S100P expression in gallbladder cancer. It was therefore suggested that LASP-1 might play a significant role in regulating metastasis of gallbladder cancer by down-regulating S100P via the PI3K/AKT pathway. It's the first study unraveling a link between LPXN and the PI3K/AKT signaling pathway. However, further studies are needed to further investigate the molecular mechanism by which LPXN regulates PI3K/AKT signaling in bladder cancer progression.

In summary, our results from in vitro and in vivo experiments provide insights into the biological function of LPXN in bladder cancer and demonstrate that LPXN overexpression 
activates the PI3K/AKT signaling pathway, leading to enhanced bladder cancer proliferation and invasion. Consequently, our study suggests that the LPXN /S100P axis and associated PI3K/AKT signaling pathways may lead to new therapeutic strategies for the with bladder cancer treatment.

\section{Acknowledgements}

The study was supported by the National Natural Science Foundation of China (Grant No. 81402303; No. 81772714).

\section{Disclosure Statement}

The authors declare to have no competing interests.

\section{References}

1 Chen W, Zheng R, Baade PD, Zhang S, Zeng H, Bray F, Jemal A, Yu XQ He J: Cancer statistics in China, 2015 CA Cancer J Clin 2016;66:115-132.

- Bellmunt J, Orsola A, Leow JJ, Wiegel T, De Santis M, Horwich A, Group EGW: Bladder cancer: ESMO Practice Guidelines for diagnosis, treatment and follow-up. Ann Oncol 2014;25 Suppl 3:iii40-48.

-3 Lipsky BP, Beals CR, Staunton DE: Leupaxin is a novel LIM domain protein that forms a complex with PYK2 J Biol Chem 1998;273:11709-11713.

4 Chen PW, Kroog GS: Leupaxin is similar to paxillin in focal adhesion targeting and tyrosine phosphorylation but has distinct roles in cell adhesion and spreading. Cell Adh Migr 2010;4:527-540.

$>5$ Gupta A, Lee BS, Khadeer MA, Tang Z, Chellaiah M, Abu-Amer Y, Goldknopf J, Hruska KA: Leupaxin is a critical adaptor protein in the adhesion zone of the osteoclast. J Bone Miner Res 2003;18:669-685.

6 Sundberg-Smith LJ, DiMichele LA, Sayers RL, Mack CP, Taylor JM: The LIM protein leupaxin is enriched in smooth muscle and functions as an serum response factor cofactor to induce smooth muscle cell gene transcription. Circ Res 2008;102:1502-1511.

7 Kaulfuss S, Grzmil M, Hemmerlein B, Thelen P, Schweyer S, Neesen J, Bubendorf L, Glass AG, Jarry H, Auber $\mathrm{B}$, Burfeind P: Leupaxin, a novel coactivator of the androgen receptor, is expressed in prostate cancer and plays a role in adhesion and invasion of prostate carcinoma cells. Mol Endocrinol 2008;22:1606-1621.

8 Kaulfuss S, von Hardenberg S, Schweyer S, Herr AM, Laccone F, Wolf S, Burfeind P: Leupaxin acts as a mediator in prostate carcinoma progression through deregulation of p120catenin expression. Oncogene 2009;28:3971-3982.

-9 Kaulfuss S, Herr AM, Buchner A, Hemmerlein B, Gunthert AR, Burfeind P: Leupaxin is expressed in mammary carcinoma and acts as a transcriptional activator of the estrogen receptor alpha. Int J Oncol 2015;47:106-114.

10 Abe A, Yamamoto Y, Iba S, Kanie T, Okamoto A, Tokuda M, Inaguma Y, Yanada M, Morishima S, Mizuta S, Akatsuka Y, Okamoto M, Kameyama T, Mayeda A, Emi N: ETV6-LPXN fusion transcript generated by $\mathrm{t}(11 ; 12)(\mathrm{q} 12.1 ; \mathrm{p} 13)$ in a patient with relapsing acute myeloid leukemia with NUP98-HOXA9 Genes. Chromosomes Cancer 2016;55:242-250.

11 Yang C, Zhang W, Wang L, Kazobinka G, Han X, Li B, Hou T: Musashi-2 promotes migration and invasion in bladder cancer via activation of the JAK2/STAT3 pathway. Lab Invest 2016;96:950-958.

12 Du M, Wang G, Ismail TM, Gross S, Fernig DG, Barraclough R, Rudland PS: S100P dissociates myosin IIA filaments and focal adhesion sites to reduce cell adhesion and enhance cell migration. J Biol Chem 2012;287:15330-15344.

13 Jiang H, Hu H, Lin F, Lim YP, Hua Y, Tong X, Zhang S: S100P is Overexpressed in Squamous Cell and Adenosquamous Carcinoma Subtypes of Endometrial Cancer and Promotes Cancer Cell Proliferation and Invasion. Cancer Invest 2016;34:477-488. 


\section{Cellular Physiology Cell Physiol Biochem 2018;47:2250-2260

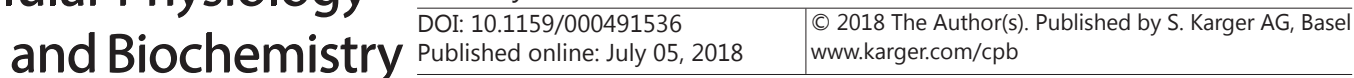

Hou et al.: LPXN Regulates BC Progression via PI3K/AKT

14 Higgins JP, Kaygusuz G, Wang L, Montgomery K, Mason V, Zhu SX, Marinelli RJ, Presti JC, Jr., van de Rijn M, Brooks JD: Placental S100 (S100P) and GATA3: markers for transitional epithelium and urothelial carcinoma discovered by complementary DNA microarray. Am J Surg Pathol 2007;31:673-680.

15 Cardone C: [Endodontic statistics: analysis of 100 cases]. Dent Cadmos 1988;56:79-80.

-16 Zhao X, Guan JL: Focal adhesion kinase and its signaling pathways in cell migration and angiogenesis. Adv Drug Deliv Rev 2011;63:610-615.

-17 Sahu SN, Nunez S, Bai G, Gupta A: Interaction of Pyk2 and PTP-PEST with leupaxin in prostate cancer cells. Am J Physiol Cell Physiol 2007;292:C2288-2296.

-18 Dierks S, von Hardenberg S, Schmidt T, Bremmer F, Burfeind P, Kaulfuss S: Leupaxin stimulates adhesion and migration of prostate cancer cells through modulation of the phosphorylation status of the actinbinding protein caldesmon. Oncotarget 2015;6:13591-13606.

19 Shi J, Wu WJ, Hu G, Yu X, Yu GS, Lu H, Yang ML, Liu B, Wu ZX: Regulation of beta-catenin transcription activity by leupaxin in hepatocellular carcinoma. Tumour Biol 2016;37:2313-2320.

20 Omura G, Ando M, Saito Y, Kobayashi K, Yoshida M, Ebihara Y, Kanaya K, Fujimoto C, Sakamoto T, Kondo $\mathrm{K}$, Asakage T, Yamasoba T: Association of the upregulated expression of focal adhesion kinase with poor prognosis and tumor dissemination in hypopharyngeal cancer. Head Neck 2016;38:1164-1169.

21 Golubovskaya VM, Ylagan L, Miller A, Hughes M, Wilson J, Wang D, Brese E, Bshara W, Edge S, Morrison C, Cance WG: High focal adhesion kinase expression in breast carcinoma is associated with lymphovascular invasion and triple-negative phenotype. BMC Cancer 2014;14:769.

-22 Calderaro J, Rebouissou S, de Koning L, Masmoudi A, Herault A, Dubois T, Maille P, Soyeux P, Sibony M, de la Taille A, Vordos D, Lebret T, Radvanyi F, Allory Y: PI3K/AKT pathway activation in bladder carcinogenesis. Int J Cancer 2014;134:1776-1784.

23 Li Z, Chen Y, Wang X, Zhang H, Zhang Y, Gao Y, Weng M, Wang L, Liang H, Li M, Zhang F, Zhao S, Liu S, Cao Y, Shu Y, Bao R, Zhou J, Liu X, Yan Y, Zhen L, Dong Q, Liu Y: LASP-1 induces proliferation, metastasis and cell cycle arrest at the G2/M phase in gallbladder cancer by down-regulating S100P via the PI3K/AKT pathway. Cancer Lett 2016;372:239-250. 